\title{
Dynamic Approximation with Feedback Control for Energy-Efficient Recurrent Neural Network Hardware
}

\author{
Jaeha Kung, Duckhwan Kim and Saibal Mukhopadhyay \\ Georgia Institute of Technology \\ 266 Ferst Drive, Atlanta, GA 30332, USA \\ \{jhkung,kimduckhwan,smukhopadhyay6\}@gatech.edu
}

\begin{abstract}
This paper presents methodology of feedback-controlled dynamic approximation to enable energy-accuracy trade-off in digital recurrent neural network (RNN). A low-power digital RNN engine is presented that employs the proposed dynamic approximation. The on-chip feedback controller is realized by utilizing hysteretic or proportional controller. The dynamic adaptation of bit-precisions during the $\mathrm{RNN}$ computation is selected as approximation approach. Considering various applications, the digital RNN engine designed in $28 \mathrm{~nm}$ CMOS shows $\sim 36 \%$ average energy saving compared to the baseline case, with only $\sim 4 \%$ of accuracy degradation on average.
\end{abstract}

\section{CCS Concepts}

- Hardware $\rightarrow$ Emerging technologies.

\section{Keywords}

Approximate computing, energy efficiency, machine learning hardware, recurrent neural network

\section{INTRODUCTION}

The convolutional neural network $(\mathrm{CNN})$ and deep learning architectures such as AlexNet [1] or GoogLeNet [2] have been widely used for complex problems like image classification and object recognition. Hardware accelerators are being investigated to improve power-efficiency of NN computations [3]. However, deep learning architectures based on feedforward neural networks are not suitable for applications with sequential data processing. A recurrent neural network (RNN), illustrated in Figure 1, is capable of dealing with systems having temporal behaviors with external input sequences. Consequently, RNN has ability to memorize the history of past inputs or states and is useful for applications that require the notion of time, for example, language modeling [4], medical diagnosis [5], human activity recognition [6] and mapping finite state machines [7], to name a few. RNN is a more general NN compared to the deep learning networks, and has great potential due to its ability to approximate nonlinear dynamical systems. The research on algorithm and application of RNN is rapidly progressing $[8,9]$, but there is relatively little effort in energy-efficient RNN hardware.

Permission to make digital or hard copies of all or part of this work for personal or classroom use is granted without fee provided that copies are not made or distributed for profit or commercial advantage and that copies bear this notice and the full citation on the first page. Copyrights for components of this work owned by others than ACM must be honored. Abstracting with credit is permitted. To copy otherwise, or republish, to post on servers or to redistribute to lists, requires prior specific permission and/or a fee. Request permissions from Permissions@acm.org.

ISLPED '16, August 08-10, 2016, San Francisco Airport, CA, USA

Copyright 2016 ACM. ISBN 978-1-4503-4185-1/16/08\$15.00

DOI: http://dx.doi.org/10.1145/2934583.2934626

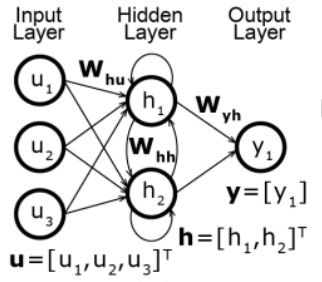

(a)

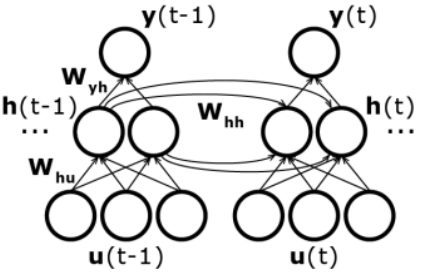

(b)
Figure 1. Overview of RNN: (a) RNN with one hidden layer where recurrent connections $\left(W_{h h}\right)$ are established between hidden neurons. (b) The same topology unfolded in time.

This paper presents the concept of feedback-controlled dynamic approximation for energy-accuracy trade-off in RNN. The approximate computing using reduced bit-precision and/or inexact arithmetic have been investigated to enable energy-accuracy trade-off in hardware accelerators for feedforward NNs [10-12]. However, to the best of our knowledge, approximate computation for digital RNN is currently unexplored. The main challenge for approximation in $\mathrm{RNN}$ is that, unlike feedforward $\mathrm{NNs}$ that operate on stationary inputs, an RNN operates on input sequences. Some parts of a given sequence may allow approximation while some parts may require precise computation. The recurrent connectivity in RNN can also lead to propagation of error over time. Hence, on-line, real-time, and dynamic control of the approximation is required in RNN. On the contrary, due to the static nature of their inputs, feedforward NNs only require static approximation [10-12]. The key contributions of this paper are:

- We develop methodology for approximate computing using dynamic adaptation of bit-precision for energy-accuracy trade-off in digital RNN.

- We present a real-time feedback controller to support the dynamic control of the approximation. The controller suppresses (encourages) approximation if output confidence gets lower (higher) than certain threshold. The hardware design of the controller is presented for faster response of the proposed algorithm.

- A digital hardware engine for RNN is presented with the integrated feedback controller to enable approximate computing (static and dynamic approximation) and, hence low-power operation. The digital RNN engine with the controller is synthesized in 28nm CMOS technology.

The algorithmic performance of the proposed feedback control is demonstrated considering human activity recognition - a wellknown benchmark in video classification [13-15]. Our analysis shows that $\sim 36 \%$ energy saving is possible using the proposed control method at the expense of $\sim 4 \%$ degradation in recognition accuracy. 


\section{PRELIMINARIES}

\subsection{Recurrent Neural Network}

Recurrent neural network (RNN) is the network where states of hidden neurons (the neurons in a hidden layer) evolve over time. A RNN is designed by additional recurrent connections (forming any cycles in the network) in a feedforward neural network, such as multilayer perceptron [16]. There are various types of RNNs depending on where recurrent connections are established. The following dynamics define the standard RNN where recurrent connections are formed from outputs of neurons in a hidden layer to their inputs (Figure 1(a)).

$$
\begin{gathered}
\boldsymbol{h}(\mathrm{t})=\mathbf{W}_{\boldsymbol{h} \boldsymbol{h}} \cdot \sigma(\boldsymbol{h}(\mathrm{t}-1))+\mathbf{W}_{\boldsymbol{h} \boldsymbol{u}} \cdot \boldsymbol{u}(\mathrm{t})+\boldsymbol{b}_{\boldsymbol{h}} \\
\boldsymbol{y}(\mathrm{t})=\mathbf{W}_{\boldsymbol{y} \boldsymbol{h}} \cdot \sigma(\boldsymbol{h}(\mathrm{t}))+\boldsymbol{b}_{\boldsymbol{y}}
\end{gathered}
$$

where $\boldsymbol{u}(t), \boldsymbol{h}(\mathrm{t}), \boldsymbol{y}(\mathrm{t})$ are inputs, states of hidden neurons and outputs at time t, respectively; $\mathbf{W}_{\boldsymbol{h u}}, \mathbf{W}_{\boldsymbol{h} \boldsymbol{h}}, \mathbf{W}_{\boldsymbol{y} \boldsymbol{h}}$ represent the weight matrices; and $\boldsymbol{b}_{\boldsymbol{h}}, \boldsymbol{b}_{\boldsymbol{y}}$ are the biases. Activation function of a neuron in the hidden layer is denoted as $\sigma(\cdot)$; mostly, a sigmoid type function. Owing to the recurrence in the network, RNN is capable of approximating any dynamic nonlinear functions [17].

The operation of RNN can be easily understood by unfolding the recurrent connections in time (Figure 1(b)). By running RNN, we can predict an output vector sequence, $\boldsymbol{y}=[\boldsymbol{y}(0), \boldsymbol{y}(1), \ldots, \boldsymbol{y}(\mathrm{T})]$ for discrete-time case, with given an input vector sequence, $\boldsymbol{u}=$ $[\boldsymbol{u}(0), \boldsymbol{u}(1), \ldots, \boldsymbol{u}(\mathrm{T})]$ where $\boldsymbol{u}(k) \in \mathbb{R}^{\text {nin }}$ and $\boldsymbol{y}(k) \in \mathbb{R}^{\text {nout }}$. The goal is to minimize (total) prediction error

$$
E_{\text {tot }}=\sum_{k} E(k)=\sum_{k}\left\|\boldsymbol{y}(k)-\boldsymbol{y}^{*}(k)\right\|^{2} / 2
$$

where $\boldsymbol{y}^{*}$ is the target output in supervised learning. The error function $E_{\text {tot }}$ can be different depending on applications. Thus, the proper training of RNN has to be performed to minimize the prediction error during the inference.

\subsection{Training of Recurrent Neural Network}

Training RNN is more complex than training feedforward NN due to its ability to learn temporal information. The backpropagation through time (BPTT) [18] is a well-known training algorithm that extends the (standard) backpropagation algorithm by unfolding the temporal behavior of the RNN to form a layered feedforward $\mathrm{NN}$. The objective of BPTT is to minimize the total error ' $E_{\text {tot }}$ ' in (3) by changing weight matrices for each training epoch. In RNN training, an epoch is one input vector sequence $\boldsymbol{u}$. Each weight update is given by

$$
\Delta w_{j i}=-\eta \frac{\partial E_{t o t}}{\partial w_{j i}}
$$

where $w_{j i}$ is a connection weight from node $i$ to $j$ and $\eta$ is the learning rate. The number of layers of constructed multilayer feedforward NN linearly increases by an additional time step involved in the training. Thus, the memory space required for BPTT increases as the length of a training sequence increases, making this method mostly suitable for off-line training of RNN.

For on-line training, real-time recurrent learning (RTRL) can be performed which updates the synaptic weights while operating the network [19]. To train RNN in real-time, RTRL tries to minimize the instantaneous error ' $E(k)$ ' in (3). The RTRL approximates the gradient of total error with respect to weight changes by an instantaneous estimate of the gradient:

$$
\Delta w_{j i, k}=-\eta \frac{\partial E(k)}{\partial w_{j i}}
$$

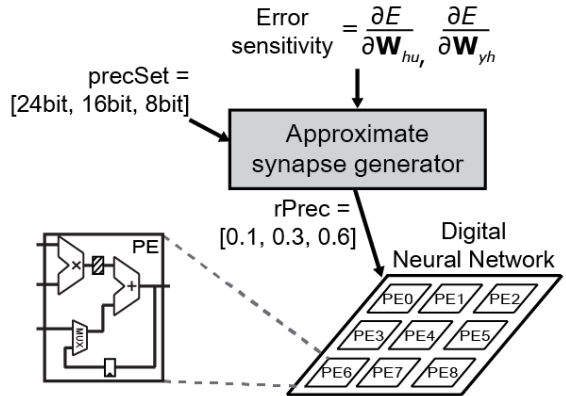

Figure 2. Overview of approximate synapse for low-power operation of digital neuromorphic hardware. Here, precSet represents the fixed bit-precision set and rPrec is the ratio of each bit-precision.

where $k$ is the current time step. The computational complexity of RTRL is high since weight update is required at every time steps.

\subsection{Approximate Feedforward Neural Network}

The recent studies have developed low-power feedforward NNs using approximate computing [10,11]. The error sensitivity of each synaptic weight $\left(\partial E / \partial w_{j i}\right)$ is computed during conventional backpropagation training of a feedforward NN. This information is utilized for the practice of approximate computing (e.g. precision control) in inference stage. As an early work regarding this topic, Venkataramani et al. minimized power by reducing bitprecisions for those neurons with lower average error sensitivity (approximate neuron) [10]. A layer with the most power consumption is selected and neurons in that layer are iteratively selected for approximation. This approach can limit the power reduction under a quality constraint as a neuron may have a few strong connections but low average error sensitivity, selecting such a neuron for approximation may limit quality.

As an extension, Kung et al. have proposed approximate synapse which is a fine-grained approach to identify synaptic weights (edges) instead of neurons in the network [11] (see Figure 2). This approach sorts entire error sensitivities and assign lower bitprecisions from a fixed bit-precision set (e.g. [24bit, 16bit, 8bit]) to synaptic connections with lower error sensitivities. A greedy algorithm was presented that starts with the least possible bitprecision (e.g. 100\% 8bit operation) and iteratively increases the ratio of higher bit-precisions until the inference of validation set reaches a given quality constraint.

\subsection{Sequence Classification using RNN}

Figure 3 illustrates the video classification (or any sequence classification) using RNN. First, an input data sequence is fed into a local feature extractor. This local feature extractor can be any type of pre-processors to identify important local features (or raw input data can be used directly). The features at each time step are passed through RNN algorithm to compute output sequences. The number of output neurons in RNN is identical to the number of video activities to classify. Each output neuron corresponds to each activity. By looking at each output vector, the maximum index at each time step ' $k$ ' is computed (by Max index identifier). Finally, the video activity is decided by selecting the one with the largest probability in the maximum index set for a pre-defined time length $\mathrm{L}$ (in Classifier). We call this time length $\mathrm{L}$ as 'decision window'. This process is continued until the input sequence ends (until $\mathrm{T}$ in Figure 3).

\section{APPROXIMATE COMPUTING IN RNN}




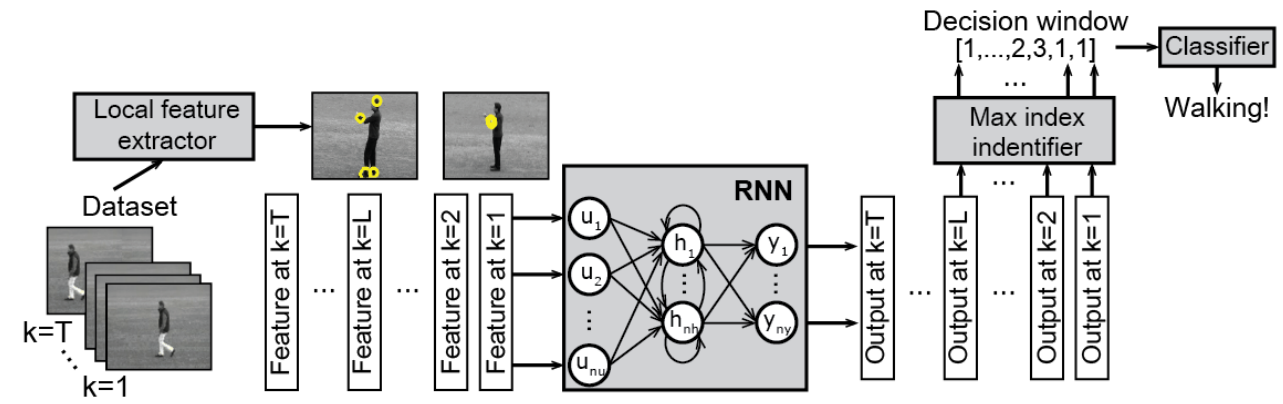

Figure 3. Block diagrams of video classification using RNN. Local feature extractor is used to recognize important local features to obtain an input sequence for RNN. Then, RNN outputs a sequence which can be post-processed to decide which activity a subject in the video is performing.

\subsection{Static Approximation in RNN}

We adopt the method of approximate synapse for RNN as the static approximation. Approximate synapse algorithm (or approximate neuron) has been presented with feedforward $\mathrm{NN}$. However, there is little effort on the application of approximate computing in RNN which naturally propagates error through time. For this reason, we evaluate static approximation in RNN as well as dynamic approximation which will be presented in the next subsection. Our approach can also utilize approximate neuron [10], however, for brevity we only consider approximate synapse.

When BPTT training algorithm is used, the only difference between the feedforward $\mathrm{NN}$ and $\mathrm{RNN}$ is an additional weight matrix $\mathbf{W}_{\boldsymbol{h} \boldsymbol{h}}$. Thus, the approximate synapse algorithm can be directly applied to RNN for computing rPrec (refer to this as static approximation). During BPTT training algorithm, the following error sensitivity vectors are computed: $\partial E_{t o t} / \partial \mathbf{W}_{h u}$, $\partial E_{t o t} / \partial \mathbf{W}_{h h}, \partial E_{t o t} / \partial \mathbf{W}_{y h}$. Then, static approximation algorithm sorts entire error sensitivities in ascending order to assign bitprecisions from low to high. As a result, it outputs $r$ Prec, e.g. [0.1, $0.3,0.6]$ when three possible bit-precisions are available.

Note the power reduction using the static approximation is limited by the choice of the input bit-precision set, e.g. [24bit, 16bit, 8bit]. The minimum required bit-precision for reasonable accuracy at the lowest power dissipation is difficult to determine a-priori and will depend on applications. Also, the feedforward NN takes stationary inputs, not a sequence. However, RNN deals with input sequences, there may be parts of the sequence that is difficult to classify with lower precision while other parts may be fairly easy. Hence, there is a need to dynamically modulate the level of approximation (in this paper, bit-precision) of each synapse over time for better power management in digital RNN.

\subsection{Dynamic Approximation in RNN}

The dynamic approximation algorithm is proposed for the energyefficient operation of digital $\mathrm{RNN}$ for sequence classification problems. It utilizes the static approximation as a basis to determine the bit-precision ratios (rPrec) and dynamically changes the bit-precision set (precSet). The proposed feedback control system is not limited to precision control but can be applied to any approximate computing methods, such as imprecise hardware or voltage over-scaling. The rPrec is kept constant for entire RNN computations during the operation of dynamic approximation. However, precSet is adaptively changed which deviates from static approximation (Figure 4).

The limitation is that static approximation uses the pre-determined bit-precision set. This set cannot be changed when each input is equally important as in the feedforward NN. In RNN, there may

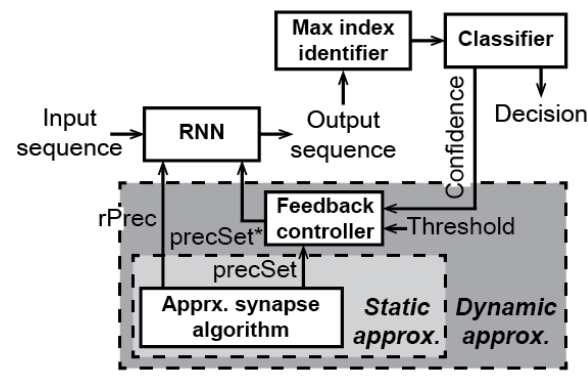

Figure 4. The proposed approximation algorithm for RNN in video (or sequence) classification.

be input sequences which are easy to detect the corresponding activity even with very low bit-precisions. On the other hand, some input sequences will make RNN to have less confidence in decision of which class (or activity) the given sequence falls in. In this scenario, reducing bit-precision may lead to decision error (accuracy degradation). Therefore, adapting precSet by looking at the current decision confidence will allow better power management in RNN. This confidence level can be checked by looking at the actual counting value, equivalently a probability mass function (PMF), of the current decision index in decision window. This is compared to pre-determined 'Threshold'. Then, the feedback controller changes precSet according to the relation between confidence level and Threshold. For instance, when the confidence is higher than the Threshold, precSet can be reduced to precSet $^{*}=$ [18bit, 10bit, 6bit] instead of using [24bit, 16bit, 8bit] (see Figure 4). The entire process of the dynamic approximation algorithm is also described in Algorithm 1. This entire process can be performed in software level. However, when the sequence that we are dealing with has faster sampling rate (such as speech), the feedback control has to be fast enough to compute next precSet $\left(\right.$ precSet $\left.^{*}\right)$ in time.

\subsection{Feedback Controller in Digital RNN}

We design and compare two different feedback controllers: 1) hysteretic controller and 2) proportional controller. A digital RNN with the feedback controller is shown in Figure 5. The dynamic precision control is done sequence by sequence (discrete-time control). A hysteretic controller has two thresholds (upper and lower). The operation is quite simple: if confidence level exceeds upper threshold, bit-precisions in precSet are decreased by one. Likewise, if the confidence level is less than lower threshold, bitprecisions in precSet are increased by one. When the confidence of classifier's output is between upper and lower threshold, precSet is sustained as it is. 


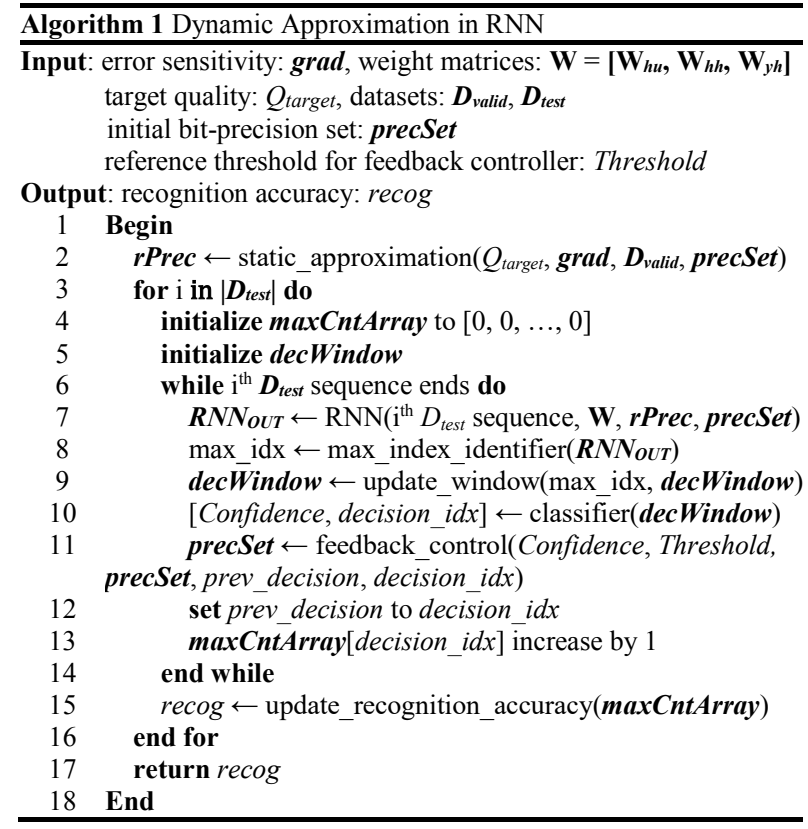

Another possible controller is a proportional controller. Hysteretic controller has a drawback when the confidence level bounces back and forth near upper (or lower) threshold which may lead to increase in switching power. The proportional controller changes precSet depending on difference between a given threshold and confidence level. The number of bits to be changed in the precSet is defined as follows:

$$
\begin{gathered}
\text { diff }=\text { Threshold }- \text { Confidence, } \\
\Delta \text { bits }=\lfloor\alpha \cdot \text { diff }+0.5\rfloor
\end{gathered}
$$

where $\alpha$ is the proportional factor and $\Delta$ bits is the number of bits to be increased or decreased in precSet. The $\alpha$ is set to 8 for our experiments (making it power of 2 simplifies hardware by using a shifter). Due to the proportionality, the controller does not change the precSet when it reaches near the threshold.

As common for both controllers, precSet bounces back to the predefined (reasonably high) bit-precision set if the current decision differs from the previous decision. The change of decision may be caused by approximation error (due to the reduced bit-precision) or different activities detected (which is important to track with high precision). Also, there are upper and lower limits where bitprecision can reach. The upper limit may be set by hardware design specification (the maximum bit-width in hardware). The lower limit is set to keep the accuracy above a certain level.

\section{SIMULATION RESULTS}

\subsection{Benchmark: Human Activity Recognition}

To verify our method, benchmarks are briefly introduced in this subsection. To validate the effect of dynamic approximation in RNN hardware, datasets related to human activity recognition are selected as benchmarks [13-15]. KTH dataset contains 6 different human actions performed by 25 subjects in four different scenarios recorded by a camera [13]. Another dataset used in the experiment is UCFG dataset which consists of 10 different actions performed by 12 subjects in four different directions (this dataset is recorded by the ground camera) [14]. The last dataset is USCHAD in which the data is captured by using wearable sensors (3axis accelerometer and gyroscope) [15]. It contains 12 different daily human activities captured from 14 subjects with 5 trials. Each dataset is divided into three sets: training, validation and

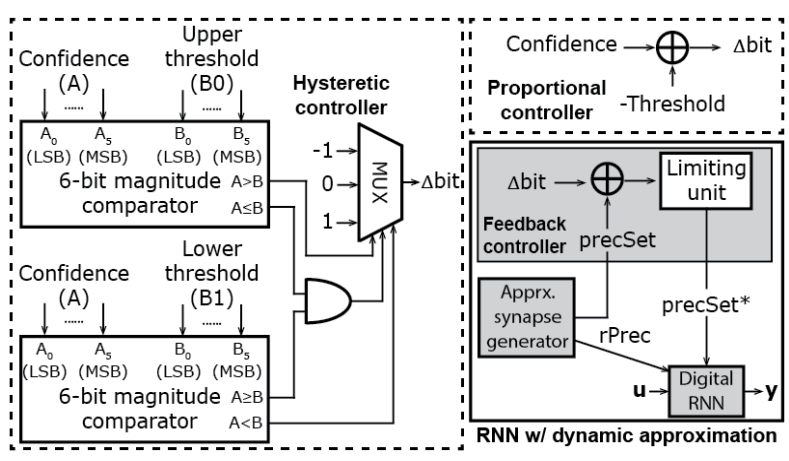

Figure 5. The proposed feedback controllers to implement dynamic approximation for RNN in hardware.

Table 1. The classification of each dataset into training, validation and inference sets

\begin{tabular}{|c|c|c|}
\hline Benchmark & Category & Subject Index \\
\hline \hline \multirow{2}{*}{$\begin{array}{c}\text { KTH } \\
(\mathbf{2 5 f p s )}\end{array}$} & Training & $11,12,14,15,16,17,18,25$ \\
\cline { 2 - 3 } & Validation & $1,4,19,20,21,23,24$ \\
\cline { 2 - 3 } & Inference & $2,3,5,6,7,8,9,10,22$ \\
\hline \multirow{2}{*}{$\begin{array}{c}\text { UCFG } \\
(\mathbf{6 0 f p s})\end{array}$} & Training & $1,2,3,4$ \\
\cline { 2 - 3 } & Validation & $5,6,7$ \\
\cline { 2 - 3 } & Inference & $8,9,10,11,12$ \\
\hline \multirow{2}{*}{$\begin{array}{c}\text { USC-HAD } \\
(\mathbf{1 0 0 H z})\end{array}$} & Training & $6,9,10,11,12$ \\
\cline { 2 - 3 } & Validation & $1,3,5,14$ \\
\cline { 2 - 3 } & Inference & $2,4,7,8,13$ \\
\hline
\end{tabular}

inference sets as summarized in Table 1. In our simulation, spacetime interest points (STIP [20]) is utilized as a local feature extractor for KTH and UCFG datasets. Since USC-HAD dataset is already measured by wearable sensors, raw data are directly used as local features. Sampling rates are also noted in Table 1. Since video sampling rate is low, both software and hardware approaches are possible for the dynamic approximation.

\subsection{Operation of Dynamic Approximation}

To simulate RNN with dynamic approximation (Figure 4), a recurrent neural network with each feedback controller is implemented using Theano library [21]. The hidden neurons are fully connected with each other. With the training set in Table 1, a RNN is trained for each dataset using BPTT training algorithm. The resulting synaptic weight matrices and error sensitivities are used in the experiment. The operations of the proposed RNN with feedback control are shown in Figure 6. In this example, a handclapping (a-d) and a boxing (e-f) video are used where each has 200 input sequences. The decision window $\mathrm{L}$ is set to 50 . A subject is performing each activity and the objective of RNN is to correctly classify the video.

Figure 6(a) and 6(b) show the operation of hysteretic controller. The upper threshold is set to 0.7 and lower threshold is set to 0.5 . Since the maximum confidence level at initial video frames (identify as boxing) exceeds the upper threshold, precSet [0] and precSet [1] are reduced by one at each sequence which are $24 \mathrm{bit}$ and 16bit initially. We do not control the minimum bit-precision (8bit) in this example. As the confidence level stays between upper and lower thresholds, bit-precisions are kept as it is. At around sequence 35 , the activity with the maximum confidence level changes from boxing to handwaving, thus the precSet sharply increases to the pre-defined bit-precisions. The bitprecision continues to change depending on the confidence level.

The same video set is used to show how proportional controller 


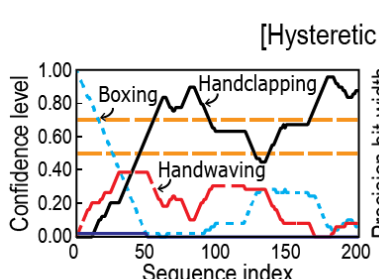

(a)

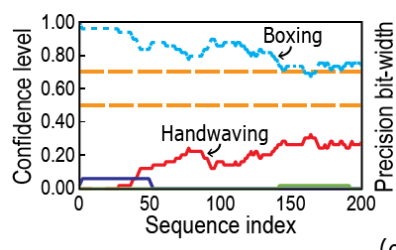

(e)

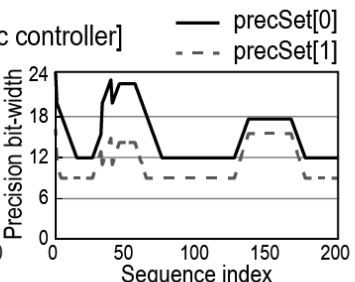

(b)

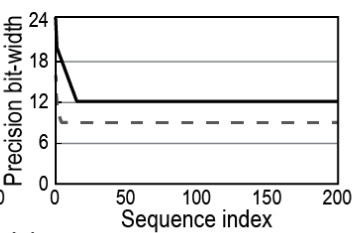

)

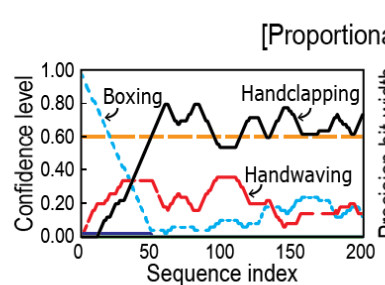

(c)

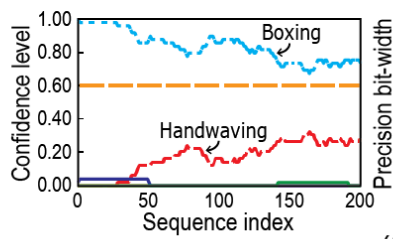

(f)

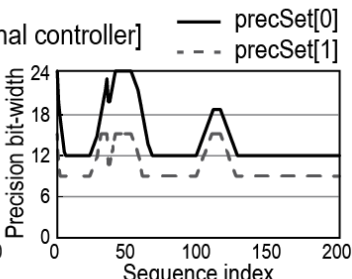

(d)

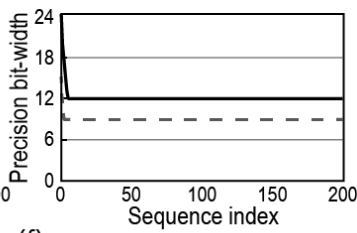

Figure 6. The operation of the dynamic approximation to adaptively change bit-precisions of synapses. (a) Confidence level of each activity and (b) the resulting precSet by using hysteretic controller. (c) Confidence level of each activity and (d) the resulting precSet by using proportional controller (handclapping). (e-f) The same set of experiment for different human activity (boxing).

Table 2. Power consumption and area breakdown of the proposed RNN hardware with dynamic approximation

\begin{tabular}{|c|c|c|c|c|}
\hline & 16 PEs & $\begin{array}{c}\text { Data CTRL } \\
\text { (Hyst, Prop) }\end{array}$ & $\begin{array}{c}\text { Max Index } \\
\text { Identifier }\end{array}$ & Classifier \\
\hline \hline \multirow{2}{*}{$\begin{array}{c}\text { Power } \\
(\mathbf{m W})\end{array}$} & 40.48 & $0.131,0.128$ & 0.596 & 0.539 \\
\cline { 2 - 5 } & $96.97 \%$ & $\mathbf{. 3 1}, \mathbf{3 1 \%}$ & $1.43 \%$ & $1.29 \%$ \\
\hline $\begin{array}{c}\text { Area } \\
\left(\boldsymbol{\mu m}^{2}\right)\end{array}$ & 70053.92 & 1077,964 & 4194.19 & 3914.17 \\
\cline { 2 - 5 } & $88.41 \%$ & $\mathbf{1 . 3 6 , 1 . 2 2 \%}$ & $5.29 \%$ & $4.94 \%$ \\
\hline
\end{tabular}

modifies precSet [Figure 6(c) and (d)]. Initially, as in the hysteretic controller, precSet decreases due to high confidence level. The difference is that precSet reaches the minimum set faster than hysteretic controller. This comes from the proportionality as in (6). It is also true when bit-precisions are required to be increased. Since hysteretic controller does not change bit-precision within the threshold band, the confidence level can go below the lower threshold. However, proportional controller keeps the confidence level near threshold quite well (Figure 6(c)). The same experiments on boxing activity shows that the feedback controller keeps bit-precision set at the lower limit when the input sequence is quite easy to classify which shows why the dynamic approximation is beneficial [6(e) and (f)].

\subsection{Digital RNN with Dynamic Approximation}

This section presents the design of the digital RNN engine with a feedback controller, synthesized in 28nm CMOS technology [22]. In digital neuromorphic hardware, the most power-consuming block is the processing engine (PE) consisting of a multiply-andaccumulate (MAC) unit and a data controller. For reasonable performance of RNN accelerator, 16 PEs are assumed in the system where they can handle MAC computations in parallel. The bit-width of PE is designed to be 24bit and 20 LSBs can be controlled to force zeros depending on the bit-precision of each computation (see inset of Figure 7). Full-precision of a MAC unit is set to 24 bit by simulating each benchmark with different bitprecisions.

The last 2 bits in each data work as dirty bits to inform which bitprecision to be used (all simulations reflect this). The precSet* is realized by the generation of a mask to additionally force zeros to simplify hardware. The static control is possible without the feedback controller and the AND gate. It is important to compute the area/power overheads of the feedback controller compared to

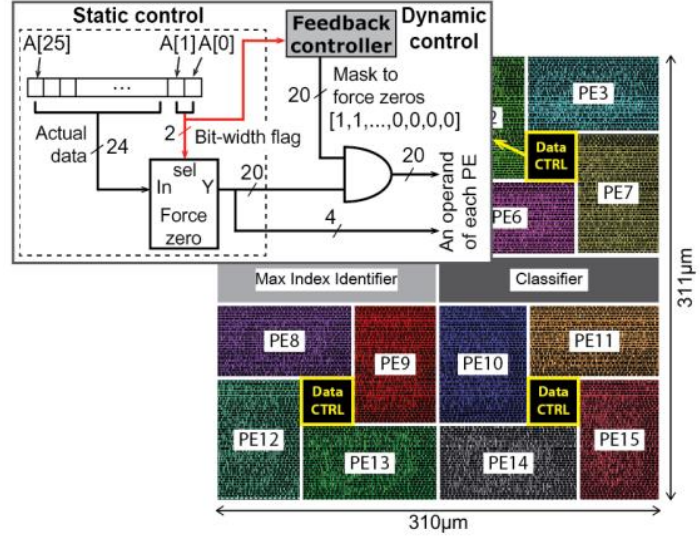

Figure 7. Synthesis and placement of the RNN hardware with dynamic approximation. A small data controller is placed near the center.

the other on-chip hardware components required to implement digital RNN with dynamic approximation. Since the dynamic approximation requires a controller per memory channel that controls data for PEs, four data controllers are designed in the system (assume 4 memory channels). The remaining components are also shown in Figure 7.

The digital RNN with dynamic approximation is integrated and synthesized in $28 \mathrm{~nm}$ technology. The power consumption and area are reported in Table 2 (prior to the placement) and the layout after the placement is shown in Figure 7. The total area is $0.096 \mathrm{~mm}^{2}$ after the placement. The most power is consumed by the PE group $(96.97 \%)$. Four data controllers, the only additional component to realize dynamic approximation, takes negligible portion $(0.31 \%)$ of power consumption. Likewise, the area of four controllers occupies only $1.22 \sim 1.36 \%$ of the total area. Even though proportional controller has smaller form factor than the hysteretic controller, both options do not impose much hardware overhead. The overhead from the static approximation is only $0.06 \mathrm{~mW}$ of power $(0.15 \%)$ and $451.3 \mu \mathrm{m}^{2}$ of area $(0.57 \%)$.

\subsection{Energy-Accuracy Trade-off}

The baseline operation of the RNN engine is with a single fixed point for all computations (e.g. 100\% 24bit). The minimum bit- 

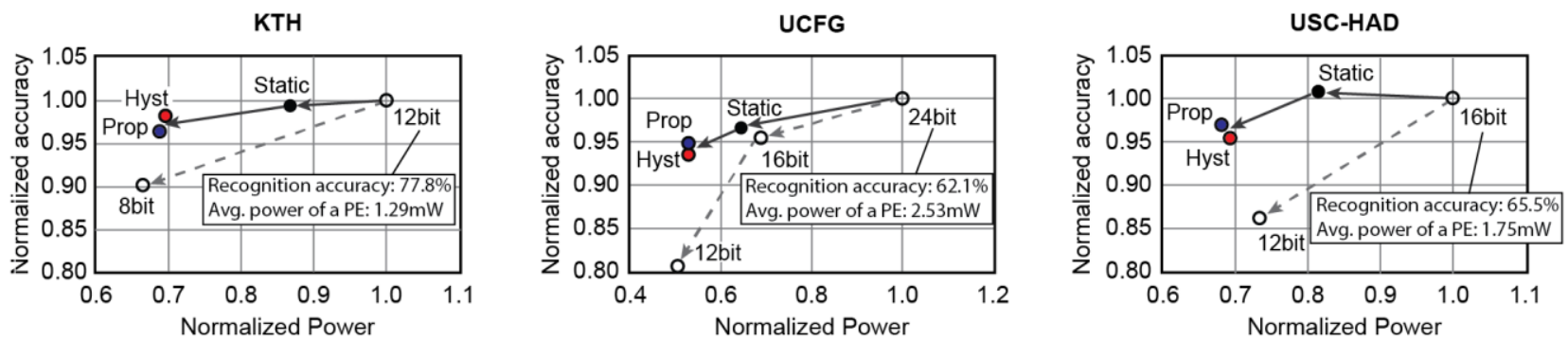

Figure 8. Simulation results showing normalized accuracy vs. normalized power for each benchmark with different computation methods: single fixed point, static approximation and dynamic approximation (hysteretic or proportional).

precision to maintain the highest possible recognition accuracy is selected for each benchmark as a baseline (refer to Figure 8). This is to make sure not to overemphasize the efficiency of the dynamic approximation algorithm. The experimental results by utilizing the static approximation algorithm in RNN is obtained as well. For both cases, the average power is computed by:

$$
P_{\text {avg }}=\sum_{i} \operatorname{rPrec}[i] \cdot P_{\text {precset }[i]}
$$

where $\operatorname{Prec}[i]$ is the $i^{\text {th }}$ precision ratio (e.g. 0.6), precSet $[i]$ is the $\mathrm{i}^{\text {th }}$ bit-precision used in the system (e.g. 18bit) and $P_{\text {precset }[i]}$ is the power dissipation of a single PE when computing with precSet $[i]$. For a RNN with dynamic approximation, the power consumption of a PE changes over time because the operating bitprecision of a PE changes dynamically. Thus, the average power consumption of single PE can be computed by:

$$
P_{\text {avg }}=\sum_{i} \operatorname{rPrec}[i] \cdot\left(\sum_{k=1}^{\mathrm{T}} P_{\operatorname{precset}(k)[i]} / \mathrm{T}\right)
$$

where $\mathrm{T}$ is the length of an input sequence and $\operatorname{precSet}(k)$ is the precSet at time step $k$.

Using the Theano simulator, recognition accuracy of each dataset is obtained by using inference dataset and results are shown in Figure 8. Compared with the baseline case, the proposed dynamic approximation degrades accuracy by $4.64 \%$ (hysteretic) and $3.93 \%$ (proportional) on average. With this slight accuracy degradation, average power consumption of a PE is reduced by $35.72 \%$ (hysteretic) and $36.36 \%$ (proportional) on average. Also, the accuracy degradation and power saving are compared with those obtained by using the static approximation. The additional power (equivalently, energy) reduction by using dynamic control is $13.82 \%$ on average while sacrificing accuracy by $3.2 \%$ on average. Note the blind control of the bit-precisions of all computations can lead to significant degradation in quality. Between two feedback control methods, a proportional controller gives slightly better performance with less power consumption. This is due to the fact that hysteretic controller stops changing bitprecision within the threshold band which may degrade accuracy as in Figure 6(a) at around sequence 140.

\section{CONCLUSIONS}

This paper presents the dynamic approximation algorithm for energy-efficient operation of digital RNN. The proposed approach adaptively controls the level of approximation in synaptic weights depending on the confidence level of output sequence. The easy sequences are computed with more approximation and hard sequences with less approximation. The dynamic control of the bit-precision is considered to verify the proposed approach. The analysis shows that the dynamic approximation allows significant power saving with graceful degradation of quality. The important future work is to apply dynamic approximation during the training of digital RNN. The proposed approach can enable application of
RNN at the sensor/camera front-ends for real-time activity detection/recognition.

\section{ACKNOWLEDGMENTS}

This material is based on work supported in parts by National Science Foundation CAREER Award and Office of Naval Research Young Investigator Program.

\section{REFERENCES}

[1] A. Krizhevsky et al., "ImageNet classification with deep convolutional neural networks," in Proc. Neural Inform. Process. Syst. (NIPS), 2012.

[2] C. Szegedy et al., "Going deeper with convolutions," in CoRR, abs/1409.4842, 2014

[3] C. Zhang et al., "Optimizing FPGA-based accelerator design for deep convolutional neural networks," in Proc. Int. Symp. Field-Programmable Gate Arrays (FPGA), 2015

[4] I. Sutskever et al., "Generating text with recurrent neural networks," in Proc. Int. Conf. Machine Learning (ICML), 2011.

[5] Z. C. Lipton et al., "Learning to diagnose with LSTM recurrent neural network," in CoRR, abs/1511.03677, 2015.

[6] M. Baccouche et al., "Sequential deep learning for human activity recognition," in Human Behavior Understanding, pp. 29-39, 2011.

[7] K. Arai et al., "Stable behavior in a recurrent neural network for a finite state machine," Neural Networks, vol. 13, no. 6, pp. 667-680, 2000.

[8] J. Chung et al., "Gated feedback recurrent neural networks," in CoRR, abs/1502.02367, 2015.

[9] W. D. Mulder et al., "A survey on the application of recurrent neural networks to statistical language modeling," Computer Speech and Language, vol. 30, no.1, pp. 61-98, 2015.

[10] S. Venkataramani et al., "AxNN: energy-efficient neuromorphic systems using approximate computing," in Proc. Int. Symp. Low Power Elec. Design (ISLPED), 2014.

[11] J. Kung et al., "A power-aware digital feedforward neural network platform with backpropagation driven approximate synapses," in Proc. Int. Symp. Low Power Elec. Design (ISLPED), 2015.

[12] S. Gupta et al., "Deep learning with limited numerical precision," in Proc. Int. Conf. Machine Learning (ICML), 2015.

[13] C. Schüldt et al., "Recognizing human actions: a local SVM approach," in Proc. Int. Conf. Pattern Recog. (ICPR), 2004.

[14] A. Nagendran, D. Harper, and M. Shah, New system performs persistent wide-area aerial surveillance, SPIE Newsroom, 2010.

[15] M. Zhang and A. A. Sawchuk, "USC-HAD: a daily activity dataset for ubiquitous activity recognition using wearable sensors," in Proc. Int. Conf. Ubiquitous Computing (UbiComp), 2012.

[16] H. Jaeger, "A tutorial on training recurrent neural networks, covering BPTT, RTRL, EKF and the "echo state network" approach," Technical Report, Oct. 2002.

[17] H. T. Siegelmann and E. D. Sontag, "Turing computability with neural nets," Applied Mathematics Letters, vol. 4, no. 6, pp. 77-80, 1991.

[18] P. J. Werbos, "Backpropagation through time: what it does and how to do it," Proc. IEEE, vol. 78, no. 10, pp. 1550-1560, 1990.

[19] R. J. Williams and J. Peng, "An efficient gradient-based algorithm for online training of recurrent network trajectories," Neural Computation, vol. 2, no. 4, pp. 490-501, 1990.

[20] I. Laptev and T. Lindeberg, "Space-time interest points," in Proc. Int Conf. Computer Vision (ICCV), 2003.

[21] F. Bastien et al., "Theano: new features and speed improvements," in Proc. Neural Inform. Process. Syst. (NIPS), 2012.

[22] "Synopsys 32/28nm generic library," https://www.synopsys.com/. 